\title{
Closed Orbit Errors from Helical Dipole Magnets
}

\author{
M. J. Syphers
}

December 22, 1995

From an "ideal" helical dipole field, where $B_{y}=B_{0} \cos k z, B_{x}=-B_{0} \sin k z$ as desired, the particle trajectory is given by

$$
\begin{aligned}
& x(s)=x_{0}-\frac{B_{0}}{(B \rho)} \frac{1}{k^{2}}(1-\cos k s)+x_{0}^{\prime} s \\
& y(s)=y_{0}+\frac{B_{0}}{(B \rho)} \frac{1}{k^{2}} \sin k s+\left(y_{0}^{\prime}-\frac{B_{0}}{(B \rho)} \frac{1}{k}\right) s
\end{aligned}
$$

where $B_{0}$ is the central dipole field, $B \rho$ is the magnetic rigidity of the particle, and the repeat period of the helical field is $L=2 \pi /|k|$. For a complete helix (i.e., for $s=L)$, these results can be written in matrix form as:

$$
\left(\begin{array}{c}
x \\
x^{\prime} \\
y \\
y^{\prime}
\end{array}\right)_{f}=\left(\begin{array}{llll}
1 & L & 0 & 0 \\
0 & 1 & 0 & 0 \\
0 & 0 & 1 & L \\
0 & 0 & 1 & 0
\end{array}\right)\left(\begin{array}{c}
x \\
x^{\prime} \\
y \\
y^{\prime}
\end{array}\right)_{\circ}+\left(\begin{array}{c}
0 \\
0 \\
-\delta \\
0
\end{array}\right)
$$

where

$$
\delta \equiv \frac{B L}{k(B \rho)}
$$

We see that a right-handed $(k>0)$ helical dipole which starts and ends with the dipole field pointed vertically upward will act like a drift space of length $L$, but produces a net vertical displacement downward by an amount $\delta$. In contrast to a "regular" dipole magnet error which can be thought of as producing a kink in the slope of the particle trajectory at the source of the error, a "helical dipole" error will introduce a step in the trajectory.

Consider a circular accelerator in which a single helical dipole magnet is suddenly turned on. The horizontal closed orbit in the accelerator will remain unchanged, while a vertical closed orbit error will be introduced. The closed orbit at the exit of the helical dipole error will thus be found by solving

$$
\left(\begin{array}{c}
y \\
y^{\prime}
\end{array}\right)_{\circ}=M\left(\begin{array}{c}
y \\
y^{\prime}
\end{array}\right)_{\circ}+\left(\begin{array}{c}
-\delta \\
0
\end{array}\right)
$$


or,

$$
\begin{aligned}
\left(\begin{array}{c}
y \\
y^{\prime}
\end{array}\right)_{\circ} & =(I-M)^{-1}\left(\begin{array}{c}
-\delta \\
0
\end{array}\right) \\
& =\frac{1}{2 \sin \pi \nu}(J \cos \pi \nu+I \sin \pi \nu)\left(\begin{array}{c}
-\delta \\
0
\end{array}\right) \\
& =\frac{-\delta}{2 \sin \pi \nu}\left(\begin{array}{c}
\alpha_{\circ} \cos \pi \nu+\sin \pi \nu \\
-\gamma_{\circ} \cos \pi \nu
\end{array}\right)
\end{aligned}
$$

where $\gamma_{\circ}=\left(1+\alpha_{\circ}^{2}\right) / \beta_{\circ}$ and $\beta_{\circ}, \alpha_{\circ}$ are the Courant-Snyder parameters at the downstream end of the helical dipole. Transporting this closed solution around the accelerator, the vertical closed orbit is then distorted according to

$$
y(s)=\sqrt{\frac{\beta(s)}{\beta_{\circ}}} \frac{\delta}{2 \sin \pi \nu}\left\{\sin [\psi(s)-\pi \nu]-\alpha_{\circ} \cos [\psi(s)-\pi \nu]\right\}
$$

where the phase advance $\psi$ is measured from the downstream end of the helical dipole.

The maximum orbit distortion will occur at a maximum amplitude function location $(\hat{\beta})$ where the phase advance happens to be $\tan (\psi-\pi \nu)=-1 / \alpha_{\circ}$, and so the maximum distortion can be estimated to be

$$
y_{\max } \approx \sqrt{\frac{\hat{\beta}}{\beta_{\circ}}} \frac{|\delta|}{2|\sin \pi \nu|} \sqrt{1+\alpha_{\circ}^{2}}=\frac{\sqrt{\hat{\beta} \gamma_{\circ}}}{2|\sin \pi \nu|}|\delta|
$$

\section{Strength/Length Errors}

As an example, consider a $4 \mathrm{~T}, L=2.4 \mathrm{~m}$ RHIC helical dipole magnet located in the middle of a dispersion suppressor half cell where $\beta_{\circ}=30 \mathrm{~m}, \alpha_{\circ}=1.5$, and use $\nu=24.18$ and $\hat{\beta}=50 \mathrm{~m}$. If we are at $250 \mathrm{GeV}$ energy, where $B \rho=800 \mathrm{~T}-\mathrm{m}$, then

$$
y_{\max } \approx 10 \mathrm{~mm} \frac{\Delta B}{B}
$$

in the arcs. The rms orbit in the arcs (with the average being taken over both focusing and defocusing quadrupoles) is then $y_{r m s} \approx y_{\max } \sqrt{\langle\beta\rangle / 2 \hat{\beta}} \approx(5 \mathrm{~mm}) \Delta B / B$.

To keep the imperfection resonance strength below $\epsilon_{i m p}<0.05$, the rms vertical closed orbit must be kept below $y_{r m s}<0.2 \mathrm{~mm}$ at $250 \mathrm{GeV}{ }^{1}$ The field in a single $4 \mathrm{~T}$ Snake magnet would have to change by $4 \%$ to generate a vertical closed orbit of this magnitude at high energy. The RHIC Snake system is aided by the fact that helical dipoles are powered in pairs of opposite polarity. Thus, the relative values

\footnotetext{
${ }^{1}$ K. Brown, et al., "Conceptual Design for the Acceleration of Polarized Protons in RHIC," May 1993, rev. Feb. 1995.
} 
of each pair of helical dipoles will need to be maintained below this level. This places requirements on the power supply system and controls system, where pairs of magnets must be regulated, as well as on the relative magnetic lengths of the pairs of magnets. However, this level of accuracy is very easily achievable. In addition, the dipole correction magnets around the accelerator will be used to smooth the orbit after the Snakes are turned on.

In the case of the spin Rotators, pairs have the same polarity field, but opposite helicity. So, in that regard, the Rotator pairs have the same relative accuracy requirements as the Snakes. However, the Rotator magnets have helical fields which begin and end horizontally. Thus, setting errors in the Rotators will generate horizontal orbit errors, which will not contribute to the imperfection resonance strengths.

\section{Rotational Alignment Errors}

For the Rotators, another source of orbit distortion would be more important to control, namely rotational alignment of the helical magnets. If a Rotator magnet is itself rotated about its longitudinal axis by an angle $\alpha$, then the effect on the particle trajectory is just

$$
\left(\begin{array}{c}
x \\
x^{\prime} \\
y \\
y^{\prime}
\end{array}\right)_{f}=\left(\begin{array}{llll}
1 & L & 0 & 0 \\
0 & 1 & 0 & 0 \\
0 & 0 & 1 & L \\
0 & 0 & 1 & 0
\end{array}\right)\left(\begin{array}{c}
x \\
x^{\prime} \\
y \\
y^{\prime}
\end{array}\right)+\left(\begin{array}{c}
\delta \cos \alpha \\
0 \\
\delta \sin \alpha \\
0
\end{array}\right)
$$

leading to a residual distortion in the vertical orbit as well as in the horizontal. So here, rotational alignment errors will generate vertical orbit distortions which can contribute to the imperfection resonances. In addition, the Rotators would most likely be adiabatically turned on after the final collision energy is reached, so that control of the vertical orbit during this operation will be important.

If the Rotators have rms relative rotation angles of $\alpha_{r m s}$ within a group of $N=4$ magnets, then the rms closed orbit in the arcs due to this group will be approximately

$$
\begin{aligned}
y_{r m s} & \approx \frac{1}{\sqrt{2}} y_{\max } \sqrt{\frac{\langle\beta\rangle}{\hat{\beta}}} \\
& =\frac{1}{\sqrt{2}} \sqrt{\langle\beta\rangle \gamma_{\circ}} \frac{|\delta| \alpha_{r m s}}{2|\sin \pi \nu|} \sqrt{N} .
\end{aligned}
$$

In the rotators, all magnets run at about $3 \mathrm{~T}$ and so $\delta=3 \mathrm{~mm}$ at $250 \mathrm{GeV}$. With $\langle\beta\rangle \gamma_{\circ} \approx 1+\left\langle\alpha_{\circ}\right\rangle^{2}$, we find that a vertical $\mathrm{rms}$ orbit distortion of $0.2 \mathrm{~mm}$ corresponds to an rms rotation angle of $\alpha=28 \mathrm{mr}$. If we have four sets of Rotators which happen to affect the orbit coherently, then that says the relative rotational alignment within each set of four magnets should be roughly $7 \mathrm{mr}$. Over a magnet yoke diameter of 14 in., this corresponds to a transverse alignment error of $7 \mathrm{in} . \times 7 \mathrm{mr}=50 \mathrm{mil}$, or $\approx 1 \mathrm{~mm}$ which is easily accomodated. 


\section{Integrated Pitch Errors}

Lastly, we consider the effect of a magnet which is built where the value of $k \times L$ is not equal to $2 \pi$. Suppose that in reality, $k L=2 \pi+2 \epsilon$. We assume that if this effect were measured after the construction of the magnet, one would attempt to install the magnet with its field oriented by $-\epsilon$ at the entrance and $+\epsilon$ at the exit of the magnet. For a helical dipole with the field oriented vertically plus an angle $\alpha$ at the entrance, the solution for the particle trajectory is

$$
\begin{aligned}
& x(s)=x_{0}+\frac{B_{0}}{(B \rho)} \frac{1}{k^{2}}[\cos (k s+\alpha)-\cos \alpha]+\left(x_{0}^{\prime}+\frac{B_{0}}{(B \rho)} \frac{1}{k} \sin \alpha\right) s \\
& y(s)=y_{0}+\frac{B_{0}}{(B \rho)} \frac{1}{k^{2}}[\sin (k s+\alpha)-\sin \alpha]+\left(y_{0}^{\prime}-\frac{B_{0}}{(B \rho)} \frac{1}{k} \cos \alpha\right) s
\end{aligned}
$$

and so if $\alpha=-\epsilon$ and $k L=2 \pi+2 \epsilon$, then

$$
\begin{aligned}
x(L) & =x_{0}+x_{0}^{\prime} L-\frac{B_{0} L}{k(B \rho)} \sin \epsilon \\
x^{\prime}(L) & =x_{0}^{\prime}-2 \frac{B_{0}}{k(B \rho)} \sin \epsilon \\
y(L) & =y_{0}+y_{0}^{\prime} L-\frac{B_{0} L}{k(B \rho)} \cos \epsilon+2 \frac{B_{0}}{(B \rho)} \frac{1}{k^{2}} \sin \epsilon \\
y^{\prime}(L) & =y_{0}^{\prime}
\end{aligned}
$$

Therefore, at the exit of the magnet the trajectory is given an "additional" step of

$$
\Delta\left(\begin{array}{c}
x \\
x^{\prime}
\end{array}\right)=\left(\begin{array}{c}
-\delta \epsilon \\
-2 \frac{\delta \epsilon}{L}
\end{array}\right), \Delta\left(\begin{array}{c}
y \\
y^{\prime}
\end{array}\right)=\left(\begin{array}{c}
2 \delta \epsilon / \pi \\
0
\end{array}\right)
$$

to first order in $\epsilon$. Here, $\delta$ is the ideal value, if $k L$ were indeed equal to $2 \pi$.

A set of Snake magnets would generate a vertical closed orbit in a similar way as did the Rotators in the example above. The two 4 Tesla magnets would dominate, and so the $28 \mathrm{mr}$ requirement found previously for one set of misaligned Rotator magnets becomes for this case $2 \epsilon / \pi=(3 \mathrm{~T} / 4 \mathrm{~T}) \times \sqrt{4 / 2} \times 28 \mathrm{mr}=30 \mathrm{mr}$, or $2 \epsilon=$ $90 \mathrm{mr}$. For two sets of Snake magnets adding coherently, this becomes a tolerance of about $k L=2 \pi \pm 45 \mathrm{mr}$, or $\pm 2.5^{\circ}$.

Rotator magnets, on the other hand, would affect the vertical closed orbit in a more complicated way. In this case, both the slope and position of the trajectory are changed. We must then solve the closed orbit equation again:

$$
\left(\begin{array}{c}
y \\
y^{\prime}
\end{array}\right)_{\circ}=(I-M)^{-1}\left(\begin{array}{c}
-\delta \epsilon \\
-2 \frac{\delta \epsilon}{L}
\end{array}\right)
$$

which, after a little algebra, reduces to

$$
y(s)=\sqrt{\frac{\beta(s)}{\beta_{\circ}}} \frac{\delta \epsilon}{2 \sin \pi \nu}\left\{\sin [\psi(s)-\pi \nu]-\left(\alpha_{\circ}+2 \frac{\beta_{\circ}}{L}\right) \cos [\psi(s)-\pi \nu]\right\} .
$$




\begin{tabular}{|l|l|c|c|c|}
\hline & Element & $\Delta(B L) / B L$ & $\alpha$ & $\Delta(k L)=2 \epsilon$ \\
\hline Alone: & Snake Magnet & $4 \%$ & - & $180 \mathrm{mr}$ \\
& Rotator Magnet & - & $55 \mathrm{mr}$ & $8 \mathrm{mr}$ \\
\hline Unit: & Snake & $2 \%$ & - & $90 \mathrm{mr}$ \\
& Rotator & - & $28 \mathrm{mr}$ & $4 \mathrm{mr}$ \\
\hline Ring: & 2 Snakes & $1 \%$ & - & $45 \mathrm{mr}$ \\
& 4 Rotators & - & $7 \mathrm{mr}$ & $1 \mathrm{mr}$ \\
\hline
\end{tabular}

Table 1: Requirements for field strength/length, rotation angle, and total pitch angle to maintain $0.2 \mathrm{~mm}$ rms orbit distortion at $250 \mathrm{GeV}$.

The rms orbit distortion in the arcs due to $N=4$ rotator magnets will then be

$$
y_{r m s} \approx \frac{|\delta| \epsilon_{r m s}}{2 \sqrt{2}|\sin \pi \nu|} \sqrt{1+\left(\alpha_{\circ}+2 \beta_{\circ} / L\right)^{2}} \sqrt{N}
$$

assuming $\beta_{\circ} \approx\langle\beta\rangle$. Putting in RHIC parameters again, this says that $(2 \epsilon)_{r m s}<4 \mathrm{mr}$ $\left(0.2^{\circ} !\right)$ to maintain an orbit distortion less than $0.2 \mathrm{~mm}$. With 4 sets of rotators in each ring, this tells us that careful orbit smoothing will need to be performed during the adiabatic turn-on of the rotator systems.

A summary of the effects discussed above is provided in Table 1 . The requirements for producing an rms orbit distortion of $0.2 \mathrm{~mm}$ at $250 \mathrm{GeV}$ are tabulated.

As with all of these specifications, there is a trade-off between the specified tolerances of the magnet design and the level at which the effects can be corrected with the accelerator's correction systems. Simulations of the operational procedures should be performed using realistic elements in the detailed RHIC lattice prior to freezing the magnet specifications. 